\title{
Sequence-based characterization of swine leucocyte antigen alleles in commercially available porcine cell lines
}

\section{S. Ho, ${ }^{*}$ M. H. Franzo-Romain, ${ }^{*}$ Y. J. Lee,† J. H. Lee† \& D. M. Smith*}

\section{Summary}

A total of 53 alleles at five highly polymorphic swine leucocyte antigen (SLA) loci (SLA-1, SLA-3, SLA-2, SLA-DRB1, and SLA-DQB1) were identified in eight commercially available porcine cell lines (ESK-4, LLCPK1, MPK, PK13, PK15, PT-K75, SK-RST, and ST). This information is essential for the use of these cell lines to understand the role of SLA genes and proteins in swine models of transplantation, xenotransplantation, and in swine immune responses to infectious diseases and vaccines. The ready availability of these cell lines also makes them a good source of reference DNA for SLA allele typing.

The highly polymorphic swine leucocyte antigen (SLA) genes in the porcine major histocompatibility complex (MHC) have been repeatedly shown to influence swine immunological traits (Mallard et al., 1989; Madden et al., 1990), vaccine responsiveness (Rothschild et al., 1984; Lumsden et al., 1993) and tumor inheritance (Tissot et al., 1989) (reviewed in Lunney et al., 2009). Favourable production traits have also been mapped close to the SLA region (reviewed in Vaiman et al., 1998). The strong influence of the SLA complex is mostly attributable to the antigen-presenting properties of $\mathrm{MHC}$ proteins in the swine adaptive immune system and to many important genes in strong linkage (Renard et al., 2006). In addition, several studies which explored pigs as potential xenograft donors for human transplantation have documented the direct recognition of SLA antigens by human $\mathrm{T}$ cells

\footnotetext{
C. S. Ho and M. H. Franzo-Romain contributed equally to this work.

* Department of Pathology, University of Michigan, Ann Arbor, MI 48109, USA, † Division of Animal Science and Resources, College of Agriculture and Life Sciences, Chungnam National University, Daejeon 305-764, Korea

Received 5 January 2009; revised 5 January 2009; accepted 4 May 2009

Correspondence: Douglas M. Smith, Department of Pathology, University of Michigan, Ann Arbor, MI 48109, USA. Tel: + 1734 6153860; Fax: + 1734 6150688;

E-mail: dousmith@umich.edu
}

(Yamada et al., 1995; Shishido et al., 1997). Studies have also shown that the SLA proteins can inhibit human natural killer (NK)-mediated cytotoxicity (Kwiatkowski et al., 1999). These xeno-recognitions suggest that there is a high level of structural homology between the swine and human MHC proteins. Thus, understanding the SLA genes is crucial to the development of pigs as large animal models for human diseases and the improvement of animal health in the pig production industry.

Relative to the human MHC system, little is known about the diversity of SLA genes in the entire pig population. To better understand the extent of polymorphism and allelic architecture of the SLA system, we have sought to discover new SLA sequences from different sample sources. We report here the sequence-based characterization of alleles at five highly polymorphic SLA loci in eight porcine cell lines that are publicly available from a commercial cell line repository. The ready availability of these cell lines makes them a good source of wellcharacterized standards for SLA allele typing. In addition, these cell lines have been useful for the culture of various pig viruses. The characterization of their SLA genes would facilitate their use in studies of peptide epitopes from these viruses and may facilitate the design of effective vaccines in the context of SLA specificities.

Eight porcine cell lines, ESK-4 (embryonic kidney), LLC-PK1 (normal kidney), MPK (normal kidney), PK13 (normal kidney), PK15 (normal kidney), PT-K75 (nasal turbinate mucosa), SK-RST (normal kidney cortex) and ST (normal testis), were obtained from the American Type Culture Collection (ATCC) (Manassas, VA, USA) and cultured according to the recommended conditions. LLC-PK1 was derived from the kidney of a Hampshire pig (Hull et al., 1976). PK13 and PK15 were established from two clones derived from the PK2a line which was also originated from a Hampshire pig (Ruddle, 1961). Interferon (IFN)- $\gamma$ (R \& D Systems, Minneapolis, MN, USA) was used at a final concentration of $100 \mathrm{ng} / \mathrm{mL}$ in the PK13, PK15 and PT-K75 cell cultures for 5 days to induce SLA class II mRNA expression. Alleles of SLA-1, SLA-3, SLA-2, SLA-DRB1 and SLA-DQB1 were amplified from complementary DNA (cDNA) using the locus-specific polymerase chain reaction (PCR) primers as previously described (Lee et al., 2005; Smith et al., 2005c; Ho et al., 2006; Lee et al., 2008). These primers were positioned in 
the untranslated regions to amplify the entire coding region. Alleles of the SLA-DQA and SLA-DRA loci were not characterized in this study due to their tight linkage to the SLA-DQB1 and SLA-DRB1 loci, respectively, and the limited polymorphism of the SLA-DRA locus. A minimum of two reverse transcriptase (RT)-PCRs were performed for each locus in each cell line to confirm obtained sequence data. Betaine (Sigma-Aldrich, St. Louis, MO, USA) was used at 1-M final concentration to facilitate the amplification of SLA-DRB1 alleles in ESK-4 (Henke et al., 1997). PCR products were cloned into the pCR4BluntTOPO vector (Invitrogen, Carlsbad, CA, USA) as previously described (Ho et al., 2006) or the pSC-B vector (Stratagene, La Jolla, CA, USA) according to manufacturer's protocols. A minimum of eight clones from each locus were screened for each cell line and at least two clones for each allele were sequenced completely in the forward and reverse direction. All sequences were submitted to the GenBank database and compared with the existing alleles in the Immuno Polymorphism Database (IPD)-MHC SLA sequence database (http://www.ebi.ac.uk/ipd/mhc/ sla/; Ellis et al., 2006). Novel alleles were submitted to the SLA Nomenclature Committee of the International Society for Animal Genetics (ISAG) for name designation based on sequence similarities and phylogenetic analysis (Smith et al., 2005a,b).

A total of 53 alleles were identified at five SLA loci in the eight porcine cell lines (Table 1). Comparisons with the published SLA sequences indicated 24 alleles were novel and six alleles were confirmatory (confirmed previously designated tentative alleles). Of the novel alleles, six were not assigned into existing allele groups (i.e. designated with a provisional alphanumeric name, e.g. SLA-1*es11) due to distinctive sequence motifs, as shown in the phylogenetic analyses (IPD-MHC SLA sequence database). Seven novel alleles were found to contain single base pair (bp) nucleotide substitutions from the published SLA sequences (Table 2). These single bp differences were confirmed by successful PCRs using sequence-specific primers (PCR-SSP) directed at the polymorphic sites (results not shown) and the sequences were therefore assigned permanent numerical names as confirmed alleles. Two novel sequences detected in PT-K75 were identical to cDNA library clones SPL010035D12 (GenBank Accession no. AK237395) and SPL010037G02 (GenBank Accession no. AK237409); they were also assigned as confirmed alleles SLA-1*1301 and SLA$2 * 1001$, respectively.

\begin{tabular}{|c|c|c|c|c|c|}
\hline Cell line/locus & SLA-1 & SLA-3 & SLA-2 & SLA-DRB1 & SLA-DQB1 \\
\hline \multirow[t]{2}{*}{ ESK-4 } & es $11^{b}$ & $04 \mathrm{es} 32^{\mathrm{b}}$ & $10 \mathrm{es} 21^{\mathrm{b}}$ & 0101 & 0701 \\
\hline & $\mathrm{es} 12^{\mathrm{b}}$ & $0502^{c}$ & es $22^{b}$ & 06030 & es $51^{b}$ \\
\hline LLC-PK1 & $1101^{b}$ & 0302 & $0701^{c}$ & $1301^{c}$ & 0901 \\
\hline \multirow[t]{3}{*}{ MPK } & 0201 & 0401 & 0201 & 0201 & 0201 \\
\hline & 0701 & & $040202^{b}$ & 0901 & 0801 \\
\hline & $11 \mathrm{mp} 11^{\mathrm{b}}$ & & & & \\
\hline PK13 & 0401 & 0401 & $040201^{c}$ & 0501 & 0201 \\
\hline PK15 & 0401 & 0401 & $040201^{c}$ & 0501 & 0201 \\
\hline \multirow[t]{3}{*}{ PT-K75 } & $08 p t 13^{b}$ & $03 p t 31^{b}$ & w09pt22 & 0102 & 0303 \\
\hline & $1201^{b}$ & $0502^{c}$ & $1001^{b}$ & 0403 & 040101 \\
\hline & $1301^{b}$ & & & & \\
\hline \multirow[t]{3}{*}{ SK-RST } & $0202^{b}$ & 0101 & $10 \mathrm{sk} 21^{\mathrm{b}}$ & 0501 & $04 s k 51^{b}$ \\
\hline & $08 s k 11^{b}$ & $0502^{c}$ & $110101^{b}$ & $1102^{b}$ & 0801 \\
\hline & sk13 & & & & \\
\hline \multirow[t]{2}{*}{ ST } & $0702^{c}$ & $0402^{c}$ & $0202^{c}$ & 0101 & 0101 \\
\hline & $\mathrm{st1} 11^{\mathrm{b}}$ & 0601 & $1201^{b}$ & 0901 & 0801 \\
\hline
\end{tabular}

Table 1. Swine leucocyte antigen (SLA) alleles identified in eight commercially available porcine cell lines ${ }^{\mathrm{a}}$

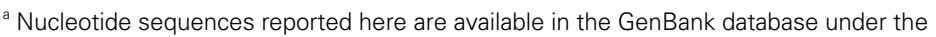
accession no. EU170457-EU170461, EU432061-EU432069, EU432072-EU432098, EU440330-EU440344, and EU496105; ${ }^{\mathrm{b}}$ novel allele; ${ }^{\mathrm{c}}$ confirmatory allele.

\begin{tabular}{|c|c|c|c|}
\hline Allele & Cell line & Similar published SLA sequence & Polymorphic position \\
\hline SLA-1*1101 & LLC-PK1 & GenBank Accession no. AJ581570 & +134 \\
\hline SLA-1*1201 & PT-K75 & GenBank Accession no. AK237712 & +457 \\
\hline SLA-1*0202 & ST & $\mathrm{SLA}-1 * 02 \mathrm{we} 02$ & +71 \\
\hline SLA-2*040202 & MPK & SLA-2*040201 & +822 \\
\hline SLA-2*110101 & SK-RST & SLA-2*110102 & $+384,+405$ \\
\hline SLA-2*1201 & ST & SLA-2*12Lw01 & +512 \\
\hline \multirow[t]{2}{*}{ SLA-DRB1*1102 } & SK-RST & SLA-DRB1*11br02 & +245 \\
\hline & & SLA-DRB1*11zs10 & +157 \\
\hline
\end{tabular}

Table 2. Novel swine leukocyte antigen (SLA) alleles with polymorphic nucleotide substitutions confirmed by PCR using sequence-specific primers (PCR-SSP)

a SLA sequence not designated by the ISAG SLA Nomenclature Committee. 
Allelic dropouts are occasionally encountered with sequence-based typing methods due to preferential PCR amplification of one allele relative to another in a heterozygous sample. This could potentially lead to the misinterpretation of the genotypes of interest. In this study, all porcine cell lines were also typed with genomic DNA by a low-resolution PCR-SSP-based typing method (Ho et al., 2009b) to confirm their SLA specificities. Briefly, this typing method relies on a set of group-specific PCR primer pairs to differentiate the published class I and class II alleles by groups with similar sequence motifs as designated by the SLA Nomenclature Committee (Smith et al., 2005a,b; Ho et al., 2009a). Despite the performance of two RT-PCRs and screening of a minimum of 20 clones for each locus, the group-specific PCR-SSP SLA typing indicated an SLA- $1 * 02 \mathrm{XX}$ group allele and an SLA-2*w09XX group allele were not detected by the sequence-based typing reactions in MPK and PT-K75, respectively (details not shown). To effectively identify these missing SLA specificities, PCR amplifications were repeated with the forward and reverse locus-specific primers independently paired with the appropriate groupspecific PCR-SSP primers (SLA-1*02XX, 5'-GCAGTT CGTGCGGTTCGACAACT-3' and 5'-GGTGTTCAGG CCCACTCGGAG-3'; SLA-2*w09XX, 5'-TGTGGGACC AGACGGGCTCT-3' and 5'-GCCTTGCAGGTAGCTCC TCCAG-3'; Ho et al., 2009b) to preferentially yield clones with overlapping sequence segments. Results revealed that the missing specificities were a confirmed allele SLA$1 * 0201$ in MPK and a novel allele SLA- $2{ }^{*}$ w09pt22 in PT-K75. Similarly, the sequence of SLA-1*08pt13 in PT-K75 was verified with the same approach of cloning overlapped sequence segments (SLA-1*08XX, 5'-CGTG GACTCCCGCTTCTTCATT-3' and 5'-GTCTCCCGAT CCCAATACTCCG-3'; Ho et al., 2009b) due to the fact that only one such full-length sequence was identified in a total of 16 clones screened.

Several SLA alleles identified in this study have unexpected sequence characteristics. An identical 3-bp deletion (codon 150; nt +510-512) in exon 3 (which encodes the $\alpha 2$ extracellular domain of the mature MHC class I protein) was observed in both SLA-3*0402 and SLA$3 * 04 \mathrm{es} 32$ in the ST and ESK-4 cell lines, respectively. In addition, SLA-3*04es 32 has a unique 12 -bp insertion in exon 4 (which encodes the transmembrane domain). A 6-bp deletion (codon 294-295; nt $+943-948$ ) in exon 5 (which encodes an intracellular domain) was observed in SLA-2*w09pt22 in the PT-K75 cell line. Additionally, the SLA-DRB1*0603Q (formerly designated SLADRB1*060202) allele detected in the ESK-4 cell line does not appear to possess the exon 5 coding region (which encodes an intracellular domain of the mature MHC class II $\beta$-chain protein). Despite having such a profound sequence modification, SLA-DRB $1 * 0603$ Q does not appear to be a sequence artefact as it has been detected independently in two porcine cDNA libraries constructed from the Landrace-Yorkshire commercial pigs (Fahrenkrug et al., 2002; Dvorak et al., 2005). Moreover, we also found multiple clones of either SLA-DRB1 allele (i.e. SLA-
DRB1*0101 and SLA-DRB1*0603Q) in ESK-4 that lack exon 5 but contain an unusual 262-bp intron 5 -like insertion between the exon 4 and exon 6 coding regions (results not shown). The addition of IFN- $\gamma$ to the cell culture and the use of betaine in locus-specific PCR did not alter or eliminate these transcripts. These unusual transcripts may have resulted from spontaneous mutations at the exonintron splice sites in subpopulations of the ESK-4 cells given that genetic instability is often observed in cultured cell lines (Maitra et al., 2005). Nevertheless, together with other SLA sequence length variants described previously (Smith et al., 2005a,b), it is not known whether the sequence modifications affect the structural integrity of the encoded SLA proteins and thus modify their surface expressions. This therefore warrants further investigation.

Five of the eight porcine cell lines characterized in this study were heterozygous across their SLA loci (with the exception at the SLA-3 locus of MPK; as explained below). Exact allele associations in the presentation of haplotypes could not be determined without further information on the SLA genotypes of the parents and/or siblings of the animals originating these cell lines. PK13 and PK15, as expected, were identical in their SLA specificities due to their derivation from the same cell line (Ruddle, 1961). Results indicated that they are homozygous for the SLA haplotype Hp-4b.5 which has been detected in the Yucatan miniature pigs (Smith et al., 2005c). LLC-PK1 was also homozygous for its SLA region which led to the designation of a new SLA haplotype $\mathrm{Hp}-25.25$ by the SLA Nomenclature Committee. Preliminary studies of SLA diversity in outbred pigs using the low-resolution PCRSSP SLA typing method indicated Hp-4b.5 and $\mathrm{Hp}-25.25$ are very common in multiple commercial pig populations (Ho et al., 2009b). Furthermore, excluding the tentative alleles without group designation, the array of SLA alleles characterized in this study correspond to 19 of the 32 class I allele groups and 15 of the 23 class II $\beta$-chain allele groups recognized to date by the SLA Nomenclature Committee (Smith et al., 2005a,b; Ho et al., 2009a). This demonstrates the porcine cell lines studied here represent a very diverse immunogenetic background.

More than two SLA-1 alleles were detected in MPK, PT-K75, and SK-RST. This suggests that the SLA haplotypes present in these cell lines have a duplicated SLA-1 locus, which has been observed in a number of class I haplotypes from multiple pig breeds (Lee et al., 2005; Smith et al., 2005b; Ho et al., 2006; 2009a; Soe et al., 2008). A recent study by Tanaka-Matsuda et al. also revealed multiple duplication events in two newly designated SLA class I haplotypes, in which as many as nine class I genes were detected at the mRNA level in one haplotype (Tanaka-Matsuda et al., 2008). On the other hand, only one SLA-3 allele was detected in the MPK cell line despite the sequencing of 22 clones. SLA allele association suggests MPK was originated from a pig that had the SLA haplotype Hp-2.2 found in the Sinclair, Hanford and NIH miniature pigs, in which no expressed SLA-3 gene was detected (Sullivan et al., 1997; Smith et al., 2005c). The sole SLA-3 sequence identified in 
MPK was therefore most likely associated with the other undesignated haplotype. Taken together, growing evidence suggests the number of expressed SLA class I loci is highly haplotype dependent.

In summary, we have successfully characterized the alleles at five polymorphic SLA loci in eight commercially available porcine cell lines. The array of alleles represented by these cell lines furthers our understanding of allelic architecture and the extent of polymorphism in the SLA system. These publicly accessible cell lines also correspond to a diverse immunogenetic background which may facilitate and expand their use in a variety of biomedical and agricultural research, e.g. in elucidating the roles of SLA proteins as immunologic barriers in pigto-human xenotransplantation as well as their influence in many important swine immune traits such as disease resistance and vaccine response.

\section{Acknowledgements}

Y. J. Lee was supported by the Korea Research Foundation (grant no. KRF-2006-612-F00002). The authors wish to thank Lauren Winfree for expert technical assistance.

\section{References}

Dvorak, C.M., Hyland, K.A., Machado, J.G., Zhang, Y., Fahrenkrug, S.C. \& Murtaugh, M.P. (2005) Gene discovery and expression profiling in porcine Peyer's patch. Veterinary Immunology and Immunopathology, 105, 301.

Ellis, S.A., Bontrop, R.E., Antczak, D.F., Ballingall, K., Davies, C.J., Kaufman, J. et al. (2006) ISAG/IUIS-VIC Comparative MHC Nomenclature Committee report, 2005. Immunogenetics, 57, 953.

Fahrenkrug, S.C., Smith, T.P., Freking, B.A., Cho, J., White, J., Vallet, J. et al. (2002) Porcine gene discovery by normalized cDNA-library sequencing and EST cluster assembly. Mammalian Genome, 13, 475 .

Henke, W., Herdel, K., Jung, K., Schnorr, D. \& Loening, S.A. (1997) Betaine improves the PCR amplification of GC-rich DNA sequences. Nucleic Acids Research, 25, 3957.

Ho, C.S., Rochelle, E.S., Martens, G.W., Schook, L.B. \& Smith, D.M. (2006) Characterization of swine leukocyte antigen polymorphism by sequence-based and PCR-SSP methods in Meishan pigs. Immunogenetics, 58, 873.

Ho, C.S., Lunney, J.K., Ando, A., Rogel-Gaillard, C., Lee, J.H., Schook, L.B. \& Smith, D.M. (2009a) Nomenclature for factors of the SLA system, update 2008. Tissue Antigens, 73, 307

Ho, C.S., Lunney, J.K., Franzo-Romain, M.H., Martens, G.W., Lee, Y.J., Lee, J.H., Wysocki, M., Rowland, R.R.R. \& Smith, D.M. (2009b) Molecular characterization of swine leukocyte antigen (SLA) class I genes in outbred pig populations. Animal Genetics, doi: 10.1111/j.1365-2052.2009.01860.x.

Hull, R.N., Cherry, W.R. \& Weaver, G.W. (1976) The origin and characteristics of a pig kidney cell strain, LLC-PK. In Vitro, 12, 670.

Kwiatkowski, P., Artrip, J.H., John, R., Edwards, N.M., Wang, S.F., Michler, R.E. \& Itescu, S. (1999) Induction of swine major histocompatibility complex class I molecules on porcine endothelium by tumor necrosis factor-alpha reduces lysis by human natural killer cells. Transplantation, 67, 211.

Lee, J.H., Simond, D., Hawthorne, W.J., Walters, S.N., Patel, A.T., Smith, D.M., O'Connell, P.J. \& Moran, C. (2005) Characterization of the swine major histocompatibility complex alleles at eight loci in Westran pigs. Xenotransplantation, 12, 303.

Lee, Y.J., Cho, K.H., Kim, M.J., Smith, D.M., Ho, C.S., Jung, K.C. et al. (2008) Sequence-based characterization of the eight SLA loci in Korean native pigs. International Journal of Immunogenetics, 35, 333.

Lumsden, J.S., Kennedy, B.W., Mallard, B.A. \& Wilkie, B.N. (1993) The influence of the swine major histocompatibility genes on antibody and cell-mediated immune responses to immunization with an aromatic-dependent mutant of Salmonella typhimurium. Canadian Journal of Veterinary Research, 57, 14.

Lunney, J.K., Ho, C.S., Wysocki, M. \& Smith, D.M. (2009) Molecular genetics of the swine major histocompatibility complex, the SLA complex. Developmental and Comparative Immunology, 33, 362.

Madden, K.B., Murrell, K.D. \& Lunney, J.K. (1990) Trichinella spiralis: major histocompatibility complex-associated elimination of encysted muscle larvae in swine. Experimental Parasitology, $70,443$.

Maitra, A., Arking, D.E., Shivapurkar, N., Ikeda, M., Stastny, V., Kassauei, K. et al. (2005) Genomic alterations in cultured human embryonic stem cells. Nature Genetics, 37, 1099.

Mallard, B.A., Wilkie, B.N. \& Kennedy, B.W. (1989) The influence of the swine major histocompatibility genes (SLA) on variation in serum immunoglobulin (Ig) concentration. Veterinary Immunology and Immunopathology, 21, 139.

Renard, C., Hart, E., Sehra, H., Beasley, H., Coggill, P., Howe, K. et al. (2006) The genomic sequence and analysis of the swine major histocompatibility complex. Genomics, 88, 96.

Rothschild, M.F., Chen, H.L., Christian, L.L., Lie, W.R., Venier, L., Cooper, M., Briggs, C. \& Warner, C.M. (1984) Breed and swine lymphocyte antigen haplotype differences in agglutination titers following vaccination with B. bronchiseptica. Journal of Animal Science, 59, 643

Ruddle, F.H. (1961) Chromosome variation in cell populations derived from pig kidney. Cancer Research, 21, 885.

Shishido, S., Naziruddin, B., Howard, T. \& Mohanakumar, T. (1997) Recognition of porcine major histocompatibility complex class I antigens by human CD8+ cytolytic T cell clones. Transplantation, 64, 340.

Smith, D.M., Lunney, J.K., Ho, C.S., Martens, G.W., Ando, A., Lee, J.H., Schook, L., Renard, C. \& Chardon, P. (2005a) Nomenclature for factors of the swine leukocyte antigen class II system, 2005. Tissue Antigens, 66, 623.

Smith, D.M., Lunney, J.K., Martens, G.W., Ando, A., Lee, J.H., Ho, C.S., Schook, L., Renard, C. \& Chardon, P. (2005b) Nomenclature for factors of the SLA class-I system, 2004. Tissue Antigens, 65, 136.

Smith, D.M., Martens, G.W., Ho, C.S. \& Asbury, J.M. (2005c) DNA sequence based typing of swine leukocyte antigens in Yucatan miniature pigs. Xenotransplantation, 12, 481.

Soe, O.K., Ohba, Y., Imaeda, N., Nishii, N., Takasu, M., Yoshioka, G. et al. (2008) Assignment of the SLA alleles and reproductive potential of selective breeding Duroc pig lines. Xenotransplantation, 15, 390.

Sullivan, J.A., Oettinger, H.F., Sachs, D.H. \& Edge, A.S. (1997) Analysis of polymorphism in porcine MHC class I genes: alterations in signals recognized by human cytotoxic lymphocytes. Journal of Immunology, 159, 2318.

Tanaka-Matsuda, M., Ando, A., Rogel-Gaillard, C., Chardon, P. \& Uenishi, H. (2009) Difference in number of loci of swine leukocyte antigen classical class I genes among haplotypes. Genomics, 93, 261.

Tissot, R.G., Beattie, C.W. \& Amoss, M.S. Jr (1989) The swine leucocyte antigen (SLA) complex and Sinclair swine cutaneous malignant melanoma. Animal Genetics, 20, 51.

Vaiman, M., Chardon, P. \& Rothschild, M.F. (1998) Porcine major histocompatibility complex. Revue Scientifique et Technique, 17, 95.

Yamada, K., Sachs, D.H. \& DerSimonian, H. (1995) Human anti-porcine xenogeneic $\mathrm{T}$ cell response. Evidence for allelic specificity of mixed leukocyte reaction and for both direct and indirect pathways of recognition. Journal of Immunology, 155, 5249 . 